\title{
Fluid black holes with electric field
}

\author{
Inyong $\mathrm{Cho}^{\mathrm{a}}$ \\ School of Liberal Arts, Seoul National University of Science and Technology, Seoul 01811, South Korea
}

Received: 30 January 2018 / Accepted: 22 December 2018 / Published online: 18 January 2019

(C) The Author(s) 2019

\begin{abstract}
We investigate the gravitational field of static perfect-fluid in the presence of electric field. We adopt the equation of state $p(r)=-\rho(r) / 3$ for the fluid in order to consider the closed $\left(\mathrm{S}_{3}\right)$ or the open $\left(\mathrm{H}_{3}\right)$ background spatial topology. Depending on the scales of the mass, spatialcurvature and charge parameters $\left(K, R_{0}, Q\right)$, there are several types of solutions in $S_{3}$ and $H_{3}$ classes. Out of them, the most interesting solution is the Reisner-Norström type of black hole. Due to the electric field, there are two horizons in the geometry. There exists a curvature singularity inside the inner horizon as usual. In addition, there exists a naked singularity at the antipodal point in $S_{3}$ outside the outer horizon due to the fluid. Both of the singularities can be accessed only by radial null rays.
\end{abstract}

\section{Introduction}

The spatial topology of the Universe is one of the unresolved problems in cosmology. From the recent cosmic microwave background radiation data, the density fraction of the curvature is estimated as $\Omega_{k}=0.000 \pm 0.005$ (95\%, Planck $\mathrm{TT}+$ lowP + lensing $+\mathrm{BAO})[1]$. Because of the observational error, it is not possible to determine the spatial topology from the data at the current stage. Some other efforts have been made in the inflation models in the closed/open universe [2-6]. The investigation of primordial density perturbation shows that the peculiar predictions of those models are beyond the resolution of the current observational data. Therefore, one needs to consider other ways in order to catch an idea of the background spatial topology, for example, the investigation of the gravitating localized objects in different topologies.

The pure closed/open $\left(\mathrm{S}_{3} / \mathrm{H}_{3}\right)$ spatial topology is achieved by a constant matter field with the equation of state,

\footnotetext{
a e-mail: iycho@seoultech.ac.kr
}

$p=-\frac{1}{3} \rho=$ constant

where $\rho>0$ for $S_{3}$ and $\rho<0$ for $H_{3}$. The resulting metric is well known as

$d s^{2}=\mp d t^{2}+\frac{d r^{2}}{1-k r^{2} / R_{0}^{2}}+r^{2} d \Omega_{2}^{2}$,

where $k=+1 /-1$ represents $S_{3} / H_{3}$, and $\rho= \pm 3 /\left(8 \pi R_{0}^{2}\right)$. For $S_{3}$, the ranges of the radial coordinate, $0 \leq r \leq R_{0}$ and $r \geq R_{0}$, are considered separately. (We shall call the former $S_{3}$-I and the latter $S_{3}$-II.) For $S_{3}$-II, we take $g_{00}=+1$ to consider only one time coordinate.

The metric (2) is the only solution to the Einstein's equation with the matter of Eq. (1). There is no additional mass term unlike in vacuum which admits the flat Minkowski space as the massless limit of the Schwarzschild spacetime. In order to achieve a nontrivial structure such as a black hole in $\mathrm{S}_{3} / \mathrm{H}_{3}$, other type of matter than Eq. (1) needs to be introduced. Then, the $S_{3} / H_{3}$ nature will be exposed only at some place of space while a nontrivial geometry is formed elsewhere.

For the nontrivial geometrical structure that admits the inherent $\mathrm{S}_{3} / \mathrm{H}_{3}$ topology, the static fluid configuration with the equation of state $p(r)=-\rho(r) / 3$ was recently studied in Ref. [7]. It was found that there are a black-hole solution $\left(S_{3}\right.$ I, $\mathrm{S}_{3}$-II, $\left.\mathrm{H}_{3}\right)$, a nonstatic cosmological solution $\left(\mathrm{S}_{3}\right.$-II, $\left.\mathrm{H}_{3}\right)$, and a singular static solution $\left(\mathrm{H}_{3}\right)$. The nontrivial geometries of these three types of solutions are sourced by fluid. At some region of space, the signature of the $\mathrm{S}_{3} / \mathrm{H}_{3}$ topology appears (near the equator for $S_{3}$-I, near the center for $S_{3}$-II, and at the asymptotic region for $H_{3}$ ). In this sense, we interpret the nontrivial geometrical configuration as a gravitating object formed in the $S_{3} / H_{3}$ background spatial topology. This object can be considered as a large fluid object which is produced in a global universe, or a local compact object which is produced in a local $S_{3} / H_{3}$ space.

In this paper, we consider the same static fluid in Ref. [7] with the electric field in spherical symmetry. If there is only 
the electric field, the spacetime is described by the ReisnerNorström solution. If we add the constant matter of Eq. (1) to the electric field, there is no consistent static solution to the Einstein's equation. Therefore, as in Ref. [7] we consider the fluid of $p(r)=-\rho(r) / 3$. The mixture of electric field and fluid form the geometry, and we expect that the $S_{3} / H_{3}$ topology due to fluid unveils at some region of space. When the electric field is turned off, the system reduces to the fluidonly case investigated in Ref. [7]. There are some other works on the gravitating solutions for static fluids (see e.g., Refs. [8-14]).

This paper consists as following. In Sect. 2, we introduce the model and field equations. In Sect. 3, we classify the solutions and discuss the spacetime structure. In Sect. 4, we discuss the geodesic motions. In Sect. 5, we study the stability of the solutions. In Sect. 6, we conclude.

\section{Model and field equations}

We consider the electric field and the perfect fluid in static state. The static metric ansatz for spherical symmetry is given by

$d s^{2}=-f(r) d t^{2}+g(r) d r^{2}+r^{2} d \Omega_{2}^{2}$.

The energy-momentum tensor for the fluid is given by

$T_{v}^{\mu}=\operatorname{diag}[-\rho(r), p(r), p(r), p(r)]$,

and we consider the equation of state which meets the $\mathrm{S}_{3} / \mathrm{H}_{3}$ boundary condition,

$p(r)=-\frac{1}{3} \rho(r)$

The field-strength tensor for the electric field is given by

$\mathcal{F}_{\mu \nu}=\partial_{\mu} A_{\nu}-\partial_{\nu} A_{\mu}$.

We consider the static electric field only, then the vector potential is given by

$A_{\mu}=\left[A_{0}(r), 0,0,0\right]$.

Then the nonvanishing components of $\mathcal{F}_{\mu \nu}$ in Eq. (6) are

$\mathcal{F}_{01}=-\mathcal{F}_{10}=E(r)=\left[f(r) A_{0}(r)\right]^{\prime}$,

where $E(r)$ is the electric field, and the prime denotes the derivative with respect to $r$. The energy-momentum tensor for the electric field is given by

$$
\begin{aligned}
\mathcal{T}_{\nu}^{\mu} & =\mathcal{F}^{\mu \alpha} \mathcal{F}_{\nu \alpha}-\frac{1}{4} \delta_{\nu}^{\mu} \mathcal{F}_{\alpha \beta} \mathcal{F}^{\alpha \beta} \\
& =\frac{E^{2}(r)}{2 f(r) g(r)} \operatorname{diag}(-1,-1,1,1) .
\end{aligned}
$$

With the metric (3) and the energy-momentum tensors (4) and (9), the nonvanishing components of the Einstein's equation, $G_{v}^{\mu}=8 \pi\left(T_{v}^{\mu}+\mathcal{T}_{v}^{\mu}\right)$, are

$G_{0}^{0}=-\frac{1}{r^{2}}+\frac{1}{r^{2} g}-\frac{g^{\prime}}{r g^{2}}=-8 \pi\left[\rho(r)+\frac{E^{2}(r)}{2 f(r) g(r)}\right]$,

$G_{1}^{1}=-\frac{1}{r^{2}}+\frac{1}{r^{2} g}+\frac{f^{\prime}}{r f g}=8 \pi\left[p(r)-\frac{E^{2}(r)}{2 f(r) g(r)}\right]$,

$$
\begin{aligned}
G_{2}^{2} & =G_{3}^{3}=\frac{f^{\prime}}{2 r f g}-\frac{f^{\prime 2}}{4 f^{2} g}-\frac{g^{\prime}}{2 r g^{2}}-\frac{f^{\prime} g^{\prime}}{4 f g^{2}}+\frac{f^{\prime \prime}}{2 f g} \\
& =8 \pi\left[p(r)+\frac{E^{2}(r)}{2 f(r) g(r)}\right] .
\end{aligned}
$$

Since the fluid and the electric field are minimally coupled only thorough gravity, the conservation of the energymomentum tensor is satisfied individually, $\nabla_{\mu} T^{\mu \nu}=0$ and $\nabla_{\mu} \mathcal{T}^{\mu \nu}=0$, which provide the field equations,

$\rho^{\prime}+\frac{f^{\prime}}{f} \rho=0, \quad \frac{3 E^{2}}{f g}\left(\frac{E^{\prime}}{E}-\frac{f^{\prime}}{2 f}-\frac{g^{\prime}}{2 g}+\frac{2}{r}\right)=0$.

These field equations give solutions for fluid and electric field in terms of the gravitational field,

$\rho(r)=$ constant $\times f(r), \quad E(r)=$ constant $\times \frac{\sqrt{f(r) g(r)}}{r^{2}}$.

\section{Classification of solutions}

With the solutions in Eq. (14) and the equation of state (5), the Einstein Eqs. (10)-(12) are solved,

$$
\begin{aligned}
\rho(r)= & -\frac{3}{8 \pi \alpha}\left\{1 \mp \frac{2 \alpha|\beta|}{r}\left[\beta\left(r^{2}+\alpha\right)\right]^{1 / 2}\right. \\
& \left.+\frac{Q^{2}}{3}\left(\frac{1}{\alpha}+\frac{1}{2 r^{2}}\right)\right\} \\
f(r)= & \frac{\rho(r)}{\rho_{c}}, \quad g^{-1}(r)=-\frac{8 \pi}{3}\left(r^{2}+\alpha\right) \rho(r), \\
E(r)= & \frac{Q}{3 r^{2}\left[\beta\left(r^{2}+\alpha\right)\right]^{1 / 2}},
\end{aligned}
$$

where, $Q$ is the electric charge, $\alpha$ and $\beta$ are integration constants, and $\rho_{c}=-9 \beta /(8 \pi)$. The above solutions reduce to 
Table 1 Classification of solutions. The signature of $\rho_{c}$ is chosen so that $f(\chi) g(\chi)>0$

\begin{tabular}{llll}
\hline Class & $\rho(\chi)$ & $f(\chi)$ & $g(\chi)$ \\
\hline$S_{3}$-I & $\frac{3}{8 \pi R_{0}^{2}}\left[1-K \cot \chi-\frac{Q^{2}}{6 R_{0}^{2}}\left(1-\cot ^{2} \chi\right)\right]$ & $\frac{\rho(\chi)}{\rho_{c}},\left(\rho_{c}>0\right)$ & $\frac{3}{8 \pi \rho(\chi)}$ \\
$S_{3}$-II & $\frac{3}{8 \pi R_{0}^{2}}\left[1 \mp K \tanh \chi-\frac{Q^{2}}{6 R_{0}^{2}}\left(1+\tanh ^{2} \chi\right)\right]$ & $\frac{\rho(\chi)}{\rho_{c}},\left(\rho_{c}<0\right)$ & $-\frac{3}{8 \pi \rho(\chi)}$ \\
$H_{3}$ & $-\frac{3}{8 \pi R_{0}^{2}}\left[1 \mp K \operatorname{coth} \chi+\frac{Q^{2}}{6 R_{0}^{2}}\left(1+\operatorname{coth}^{2} \chi\right)\right]$ & $\frac{\rho(\chi)}{\rho_{c}},\left(\rho_{c}<0\right)$ & $-\frac{3}{8 \pi \rho(\chi)}$ \\
\hline
\end{tabular}

those of the fluid-only solutions in Ref. [7] when $Q=0$, and to the Reisner-Norström (RN) solution when $\alpha \rightarrow \infty$ and $\beta \rightarrow 0$ with $\alpha \beta=$ finite $=M^{2 / 3}$.

In order to catch the idea of the spatial topology, we transform the radial coordinate $r$ to $\chi$, and use the metric

$d s^{2}=-f(\chi) d t^{2}+g(\chi) d \chi^{2}+R_{0}^{2} b^{2}(\chi) d \Omega_{2}^{2}$,

where $b(\chi)$ is introduced in the subsections below. We introduced a new parameter $R_{0} \equiv \sqrt{|\alpha|}$ which is related with the curvature. In addition, we introduce another parameter $K \equiv 2 R_{0}^{2}|\beta|^{3 / 2}$ interpreted as a mass parameter analogous to the fluid-only black hole investigated Ref. [7]. Depending on the signatures of $\alpha$ and $\beta$, the solutions are classified into three categories. Two of them meet the $S_{3}$ boundary condition, and the other does the $\mathrm{H}_{3}$ condition. The classes are summarized in Table 1 . When both of the parameter $K$ and the charge $Q$ are turned off, the metric reduces to that of the pure $S_{3} / H_{3}$ in Eq. (2)

\section{$3.1 S_{3}-\mathrm{I}$}

This is the case of $\alpha<0$ and $\beta<0$. The transformation is performed by

$r=R_{0} b(\chi)=R_{0} \sin \chi \quad\left(0 \leq \chi \leq \pi, 0 \leq r \leq R_{0}\right)$.

Note that for a given value of $r, \chi$ is double valued. The metric becomes

$$
\begin{aligned}
d s^{2}= & -\frac{3}{8 \pi R_{0}^{2} \rho_{c}}\left[1-K \cot \chi-\frac{Q^{2}}{6 R_{0}^{2}}\left(1-\cot ^{2} \chi\right)\right] d t^{2} \\
& +\frac{R_{0}^{2}}{1-K \cot \chi-\left(Q^{2} / 6 R_{0}^{2}\right)\left(1-\cot ^{2} \chi\right)} d \chi^{2} \\
& +R_{0}^{2} \sin ^{2} \chi d \Omega_{2}^{2} .
\end{aligned}
$$

Here, $\rho_{c}>0$. This solution states that the fluid with the electric field strength in Eq. (8) closes the space in a finite region $0 \leq r \leq R_{0}$. We believe that the fluid is responsible for this closure since the same phenomenon occurs even in the fluid-only case in Ref. [7]. Both of the Ricci scalar and the Kretschmann scalar diverge at $\chi=0$ and $\pi$, i.e., there exist curvature singularities at both poles. For the pure fluid case
( $Q=0$ ) investigated in Ref. [7], the background $S_{3}$ topology is exposed at the boundary around the equator $(\chi \approx \pi / 2$, i.e., $\left.r \approx R_{0}\right)$,

$d s_{3}^{2} \approx R_{0}^{2} d \chi^{2}+R_{0}^{2} \sin ^{2} \chi d \Omega_{2}^{2}$.

With the electric field, however, there is a charge correction,

$d s_{3}^{2} \approx \frac{R_{0}^{2}}{1-Q^{2} /\left(6 R_{0}^{2}\right)} d \chi^{2}+R_{0}^{2} \sin ^{2} \chi d \Omega_{2}^{2}$.

The location of the horizon is found from $g_{\chi \chi}^{-1}=0$,

$$
\begin{gathered}
\chi_{h}=\chi_{ \pm} \equiv \cot ^{-1}\left(\frac{3 K R_{0}^{2} \mp \sqrt{J_{1}}}{Q^{2}}\right), \\
\text { where } J_{1}=9 K^{2} R_{0}^{4}-6 Q^{2} R_{0}^{2}+Q^{4} .
\end{gathered}
$$

Depending on the existence of the horizon, there are two types of solutions. (See Fig. 1 for the graphical view of the metric function.)

(i) $R N$ black-hole type solution If $J_{1}>0$, there exist two horizons at $\chi_{h}=\chi_{ \pm}$, which coalesce when $J_{1}=0$. This solution mimics the Reisner-Nordström geometry of the charge black hole. The spacetime is regular at $\chi<\chi_{-}$ and $\chi>\chi_{+}$. The singularity at the north pole $(\chi=0)$ is inside the inner horizon, and is not accessible by the timelike observers as in the RN black hole. The singularity at the south pole $(\chi=\pi)$ is naked, but is not accessible either by the timelike observers as in the fluid black hole investigated in Ref. [7]. The geodesics are studied in the next section.

(ii) Naked singular solution If $J_{1}<0$, there is no horizon. Both singularities are naked, but neither of them are accessible.

\section{$3.2 S_{3}$-II}

This is the case of $\alpha<0, \beta>0$. The transformation is performed by

$r=R_{0} b(\chi)=R_{0} \cosh \chi \quad\left(-\infty<\chi<\infty, r \geq R_{0}\right)$, 


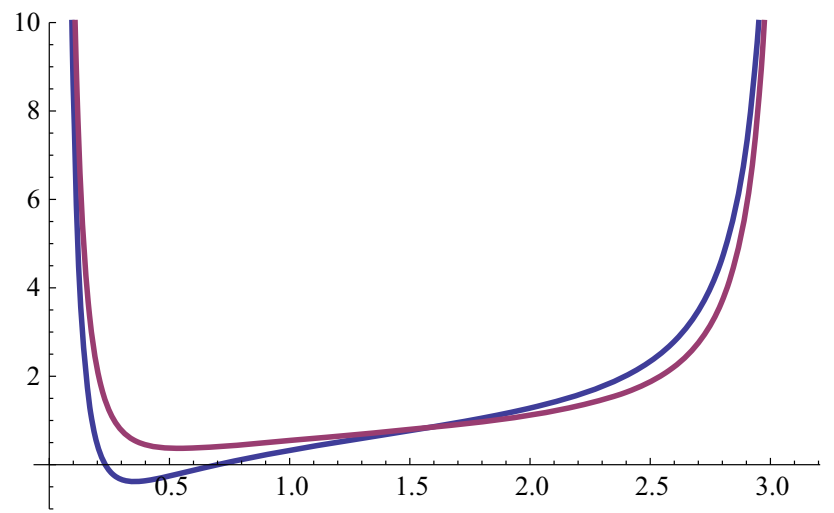

Fig. 1 Plot of metric function $8 \pi R_{0}^{2} \rho(\chi) / 3$ for $S_{3}$-I. (i) RN blackhole type solution: $K=0.9, Q=1, R_{0}=1$. There are two horizons between which the spacetime is nonstatic. There exist two curvature singularities. Neither of them is accessible except by radial null rays. (ii) Naked singular solution: $K=5 / 9, Q=1, R_{0}=1$

Again, $\chi$ is double-valued for a given value of $r$. The metric becomes

$$
\begin{aligned}
d s^{2}= & -\frac{3}{8 \pi R_{0}^{2} \rho_{c}}\left[1 \ominus \oplus K \tanh \chi-\frac{Q^{2}}{6 R_{0}^{2}}\left(1+\tanh ^{2} \chi\right)\right] d t^{2} \\
& +\frac{R_{0}^{2}}{-\left[1 \ominus \oplus K \tanh \chi-\left(Q^{2} / 6 R_{0}^{2}\right)\left(1+\tanh ^{2} \chi\right)\right]} d \chi^{2} \\
& +R_{0}^{2} \cosh ^{2} \chi d \Omega_{2}^{2} .
\end{aligned}
$$

Here, $\rho_{c}<0$. The fluid curves the space in a flipped way to the $S_{3}$-I case; the space is confined in the open region $r \geq R_{0}$. The curvature is finite everywhere. The location of the horizon is

$$
\begin{gathered}
\chi_{h}=\chi_{ \pm} \equiv \tanh ^{-1}\left(\frac{\ominus \oplus 3 K R_{0}^{2} \pm \sqrt{J_{2}}}{Q^{2}}\right), \\
\text { where } J_{2}=9 K^{2} R_{0}^{4}+6 Q^{2} R_{0}^{2}-Q^{4} .
\end{gathered}
$$

(The \pm roots are valid for both $\ominus$ and $\oplus$.) There are four types of solutions. (See Fig. 2.) Two of them are blackhole type solutions (Schwarzschild and Reisner-Nordström types) without a singularity, and the others are regular and nonstatic solutions.

Let us consider the $\ominus$ solution.

If $J_{2}>0$, there are three types of solutions.

(i) $R N$ black-hole type solution For $Q^{2} \geq 3(1+K) R_{0}^{2}$, there are two horizons at $\chi_{ \pm}$and this is the RN blackhole type.

(ii) Schwarzschild black-hole type solution For 3(1 $K) R_{0}^{2}<Q^{2}<3(1+K) R_{0}^{2}$, there exists only one horizon. Inside the horizon (the trapped region), $f(\chi), g(\chi)<0$ and $\rho>0$. The spacetime is nonstatic in the trapped region, and static outside. The structure is similar to that of the Schwarzschild black hole.

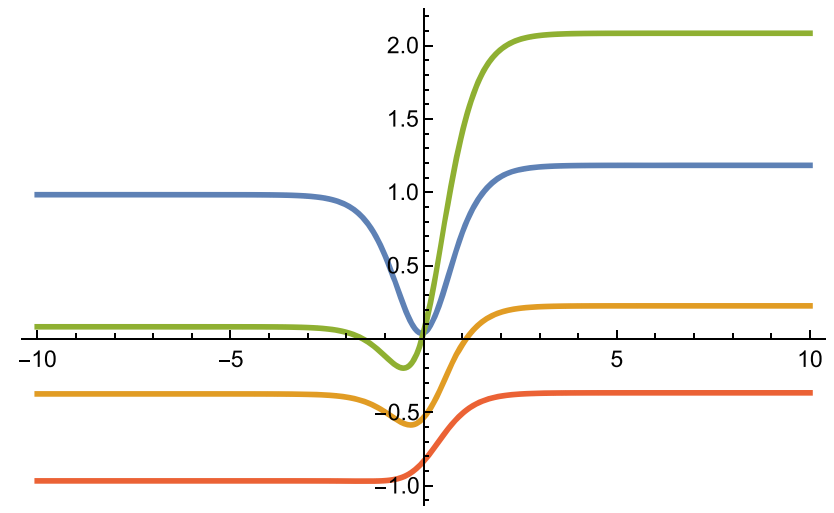

Fig. 2 Plot of metric function $-8 \pi R_{0}^{2} \rho(\chi) / 3$ for $S_{3}$-II. (i) RN blackhole type solution: $K=1, Q=1, R_{0}=0.4$. (ii) Schwarzschild black-hole type solution: $K=0.3, Q=1, R_{0}=0.6$. (iii) Nonstatic solution: $K=0.1, Q=1, R_{0}=0.4$. (iv) Regular solution: $K=0.3$, $Q=1, R_{0}=1$

(iii) Nonstatic solution For $Q^{2} \leq 3(1-K) R_{0}^{2}$, there is no horizon and the spacetime is nonstatic everywhere. This type of solution is special for $S_{3}$-II. This is analogous to the solution in Eq. (2) describing the region $r \geq$ $R_{0}$ in which the roles of the temporal and the radial coordinates are exchanged.

If $J_{2}<0$, there is one type of solution.

(iv) Regular solution The spacetime is regular everywhere while $\rho<0$.

For the $\oplus$ solution, the situation is the same with the $\ominus$ solution with $\chi \rightarrow-\chi$. Therefore, out of four types (i)-(iv), the only change is in (ii). Now, the region of $\chi<\chi_{h}$ is static, and the region of $\chi>\chi_{h}$ is nonstatic.

\section{$3.3 \mathrm{H}_{3}$}

This is the case of $\alpha>0, \beta>0$. The transformation is performed by

$r=R_{0} b(\chi)=R_{0} \sinh \chi \quad(\chi \geq 0, r \geq 0)$,

and the metric becomes

$$
\begin{aligned}
d s^{2}= & -\frac{3}{8 \pi R_{0}^{2}\left(-\rho_{c}\right)}\left[1 \ominus \oplus K \operatorname{coth} \chi+\frac{Q^{2}}{6 R_{0}^{2}}\left(1+\operatorname{coth}^{2} \chi\right)\right] d t^{2} \\
& +\frac{R_{0}^{2}}{1 \ominus \oplus K \operatorname{coth} \chi+\left(Q^{2} / 6 R_{0}^{2}\right)\left(1+\operatorname{coth}^{2} \chi\right)} d \chi^{2} \\
& +R_{0}^{2} \sinh ^{2} \chi d \Omega_{2}^{2}
\end{aligned}
$$

Here, $\rho_{c}<0$. The curvature diverges at $\chi=0$. The location of the horizon is 


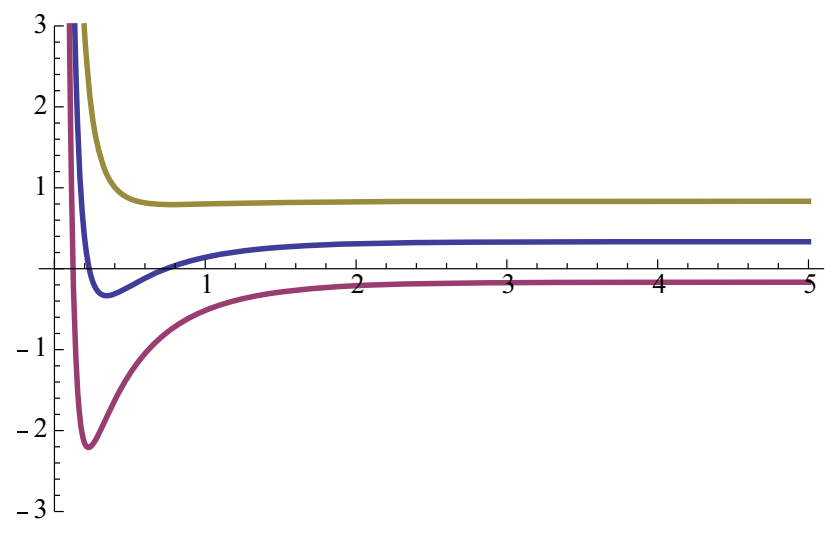

Fig. 3 Plot of metric function $-8 \pi R_{0}^{2} \rho(\chi) / 3$ for $H_{3}$. (i) RN blackhole type solution (blue): $K=1, Q=1, R_{0}=1$. (ii) dS-type solution (red): $K=1.5, Q=1, R_{0}=1$. (iii) Naked singular solution: $K=0.5$, $Q=1, R_{0}=1$

$$
\begin{gathered}
\chi_{h}=\chi_{ \pm} \equiv \operatorname{coth}^{-1}\left(\frac{\oplus \ominus 3 K R_{0}^{2} \mp \sqrt{J_{3}}}{Q^{2}}\right), \\
\text { where } J_{3}=9 K^{2} R_{0}^{4}-6 Q^{2} R_{0}^{2}-Q^{4} .
\end{gathered}
$$

The solutions are classified as below. (See Fig. 3.)

For the $\ominus$ solution in Eq. (28), there are three types of solutions for $J_{3}>0$.

(i) $R N$ black-hole type solution For $3(K-1) R_{0}^{2}<Q^{2}<$ $3 K R_{0}^{2}$, there are two horizons at $\chi_{ \pm}$and this is the $\mathrm{RN}$ black-hole type.

(ii) dS-type solution For $Q^{2} \leq 3(K-1) R_{0}^{2}$, there is only one horizon at $\chi_{+}$. The spacetime is static inside the horizon, and nonstatic outside. This is a de Sitter-like solution. This solution is achieved when the electric charge $Q$ is small. When $Q=0$, this corresponds to the cosmological solution of the fluid-only case in Ref. [7] for which the spacetime is nonstatic everywhere. It was interpreted as a universe expanding from an initial singularity. For the present case, however, the horizon is formed due to the electric field inside which the spacetime is static.

(iii) Naked singular solution For $Q^{2} \geq 3 K R_{0}^{2}$, the solution is static everywhere, but with a singularity at the center.

For the $\oplus$ solution in Eq. (28), or for $J_{3}<0$, there is no horizon, and the solution is singular static like (iii).

\subsection{Gauss' law}

Let us discuss the Gauss' law in the $\chi$ coordinate. The fieldstrength tensor $\mathcal{F}_{\mu \nu}$ in Eq. (6) in the $r$ coordinate with the components (8) is transformed to $\mathcal{F}_{\mu \nu}^{\prime}$ in the $\chi$ coordinate with the nonzero components,

$$
\mathcal{F}_{t \chi}^{\prime}=-\mathcal{F}_{\chi t}^{\prime}=E(\chi)=\frac{Q}{3|\beta|^{1 / 2} R_{0}^{2} b^{2}(\chi)} \text {. }
$$

The electric flux is then

$$
\begin{aligned}
\Phi_{E}= & \oint E \sqrt{g^{(2)}} d^{2} x=\iint \frac{Q}{3|\beta|^{1 / 2} R_{0}^{2} b^{2}(\chi)} \\
& \times R_{0}^{2} b^{2}(\chi) \sin \theta d \theta d \phi=\frac{4 \pi Q}{3|\beta|^{1 / 2}} \\
& =\frac{4 \pi Q}{\sqrt{8 \pi\left|\rho_{c}\right|}},
\end{aligned}
$$

where we used the relation $\rho_{c}=-9 \beta /(8 \pi)$. Compared with the Gauss' law in flat space, there is a correction due to fluid by the factor $\sqrt{8 \pi\left|\rho_{c}\right|}$.

\subsection{Mass}

In this section, let us discuss the mass of the black-hole solutions. For the fluid-only case in Ref. [7], it was investigated that the horizon structure of the fluid black hole is similar to that of the Schwarzschild black hole. The parameters are related with the Schwarzschild mass $M$ as

$$
\begin{gathered}
K=\left(\frac{R_{0}^{2}}{4 M^{2}}-1\right)^{-1 / 2},\left(-\frac{R_{0}^{2}}{4 M^{2}}+1\right)^{-1 / 2}, \\
\left(\frac{R_{0}^{2}}{4 M^{2}}+1\right)^{-1 / 2},
\end{gathered}
$$

for the type $S_{3}$-I, $S_{3}$-II, and $H_{3}$, respectively. For the $S_{3}$-I type, there is an upper limit in the mass, $M \rightarrow R_{0} / 2$ as $K \rightarrow \infty$. In this limit, the horizon approaches the equator of $S_{3}, \chi_{h}=\cot ^{-1}(1 / K) \rightarrow \pi / 2$.

Other than the Schwarzschild mass, it is interesting to consider the Misner-Sharp mass $\mathcal{M}$ which can be used for blackhole thermodynamics [15]. We evaluate $\mathcal{M}$ in this work. When the metric is given by

$d s^{2}=h_{a b} d x^{a} d x^{b}+r^{2}(x) d \Omega_{2}^{2}$,

where $a, b=0,1$, the Misner-Sharp mass is defined as

$\mathcal{M}=\frac{1}{2}\left(1-h^{a b} \partial_{a} r \partial_{b} r\right)$

In the $\chi$ coordinate, we have $r=R_{0} b(\chi)$ and Eq. (34) becomes

$\mathcal{M}(\chi)=-\frac{4 \pi R_{0}^{3}}{3 s} \rho(\chi) b(\chi)\left[b^{\prime}(\chi)\right]^{2}+\frac{R_{0}}{2} b(\chi)$,

where $s$ is the signature of $\rho_{c}\left(s=+1\right.$ for $S_{3}-\mathrm{I}$, and $s=-1$ for the others). The mass depends on the radial coordinate $\chi$.

For the fluid-only case $(Q=0)$, the mass is still $\chi$ dependent, while one has $\mathcal{M}_{\mathrm{Sch}}=M$ for the ordinary Schwarzschild black hole. For the fluid black-hole solutions, 
Table 2 Effective potential $V(\chi)$

\begin{tabular}{lll}
\hline Class & $F(\chi)$ & $V(\chi)$ \\
\hline$S_{3}$-I & $1-K \cot \chi-\left(Q^{2} / 6 R_{0}^{2}\right)\left(1-\cot ^{2} \chi\right)$ & $\frac{1}{2}\left[1-K \cot \chi-\left(Q^{2} / 6 R_{0}^{2}\right)\left(1-\cot ^{2} \chi\right)\right]\left(\frac{L^{2}}{\sin ^{2} \chi}+\frac{\varepsilon}{R_{0}^{2}}\right)$ \\
$S_{3}$-II & $-1 \pm K \tanh \chi+\left(Q^{2} / 6 R_{0}^{2}\right)\left(1+\tanh ^{2} \chi\right)$ & $\frac{1}{2}\left[-1 \pm K \tanh \chi+\left(Q^{2} / 6 R_{0}^{2}\right)\left(1+\tanh ^{2} \chi\right)\right]\left(\frac{L^{2}}{\cosh ^{2} \chi}+\frac{\varepsilon}{R_{0}^{2}}\right)$ \\
$H_{3}$ & $1 \mp K \operatorname{coth} \chi+\left(Q^{2} / 6 R_{0}^{2}\right)\left(1+\operatorname{coth}^{2} \chi\right)$ & $\frac{1}{2}\left[1 \mp K \operatorname{coth} \chi+\left(Q^{2} / 6 R_{0}^{2}\right)\left(1+\operatorname{coth}^{2} \chi\right)\right]\left(\frac{L^{2}}{\sinh ^{2} \chi}+\frac{\varepsilon}{R_{0}^{2}}\right)$ \\
\hline
\end{tabular}

one can show with the aid of Eq. (32) that the MisnerSharp mass evaluated on the horizon coincides with the Schwarzschild mass, $\mathcal{M}\left(\chi_{h}\right)=M$. This indicates that the horizon structure of the fluid black hole is the same with that of the Schwarzschild black hole.

For the ordinary RN black hole, the Misner-Sharp mass is given by $\mathcal{M}_{\mathrm{RN}}=M-Q^{2} /(2 r)=M-Q^{2} /\left[2 R_{0} b(\chi)\right]$. For the RN black-hole type solutions obtained in this work $(Q \neq$ 0 ), keeping the mass relation of $K$ in Eq. (32), the MisnerSharp mass evaluated on the horizons does not coincide with that of the ordinary RN black hole, $\mathcal{M}\left(\chi_{ \pm}\right) \neq \mathcal{M}_{\mathrm{RN}}\left(\chi_{ \pm}\right)$.

Although the horizon structure of the fluid black hole ( $Q=0)$ is the same with that of the ordinary one, the thermodynamics must be very different because the off-horizon structure is very different. We shall study the thermodynamics using the Misner-Sharp mass in a separate work including the charged case.

\section{Geodesics}

In this section, we discuss the geodesics of the solutions. We focus mainly on the black-hole solutions. For simplicity, we define a function,

$F(\chi) \equiv \frac{8 \pi R_{0}^{2}}{3 s} \rho(\chi)$

The geodesic equations become

$$
\begin{aligned}
& t-\text { eq. : } \frac{1}{F(\chi)} \frac{d}{d \lambda}\left[F(\chi) \frac{d t}{d \lambda}\right]=0, \\
& \phi-\text { eq. : } \frac{1}{b^{2}(\chi)} \frac{d}{d \lambda}\left[b^{2}(\chi) \frac{d \phi}{d \lambda}\right]=0 .
\end{aligned}
$$

From Eqs. (37) and (38), we denote the conserved quantities $E$ (energy) and $L$ (angular momentum) as

$$
E \equiv F(\chi) \frac{d t}{d \lambda}=\text { constant }, \quad L \equiv b^{2}(\chi) \frac{d \phi}{d \lambda}=\text { constant }
$$

The $\chi$-equation can be derived from the metric as

$g_{\mu \nu} \frac{d x^{\mu}}{d \lambda} \frac{d x^{\nu}}{d \lambda}=-\varepsilon$

where $\varepsilon=0,1$ for null and timelike geodesics, individually. On the $\theta=\pi / 2$ plane, Eq. (40) becomes

$\frac{1}{2}\left(\frac{d \chi}{d \lambda}\right)^{2}+V(\chi)=\frac{3 E^{2}}{16 \pi R_{0}^{4}\left|\rho_{c}\right|} \equiv \tilde{E}^{2}$,

where the effective potential is given by

$V(\chi)=\frac{1}{2} F(\chi)\left[\frac{L^{2}}{b^{2}(\chi)}+\frac{\varepsilon}{R_{0}^{2}}\right]$.

We summarize $V(\chi)$ in Table 2. The effective potential $V(\chi)$ of the black-hole type solutions is plotted in Figs. 4, 5 and 6.

For the RN black-hole type solution of $S_{3}$-I, the singularities at both poles are not accessible except by the radial null geodesic. For the fluid-only case in Ref. [7], the one at the north pole inside the horizon was accessible since the inner geometry was similar to that of the Schwarzschild black hole. However, for the present case, it is not because the inner geometry is similar to that of the charged black hole. The nonaccessibility to the naked singularity at the south pole is similar to the fluid-only case. The geodesic observer starting from the outer static region falls into the inner static region passing the intermediate nonstatic region. Afterwards, the observer bounces back to the nonstatic region and then enters the outer static region. This later motion after the bounce proceeds in the other copy of the spacetime accompanied in the usual RN geometry. The geodesic as a whole is an oscillatory orbit in the infinite tower of the RN spacetime.

For $S_{3}$-II, the RN black-hole type solution, when the energy level $(\tilde{E})$ is low, the oscillatory orbit is similar to that of $S_{3}$-I. When the energy level is increased, the geodesic observer can reach the inner static region behind the inner horizon. When the energy level is high enough, the geodesic observer can escape to the asymptotic infinity in the static region. The Schwarzschild black-hole type solution has the similar geodesic structure to that of the usual Schwarzschild black hole. When the energy level is low, all the geodesic 


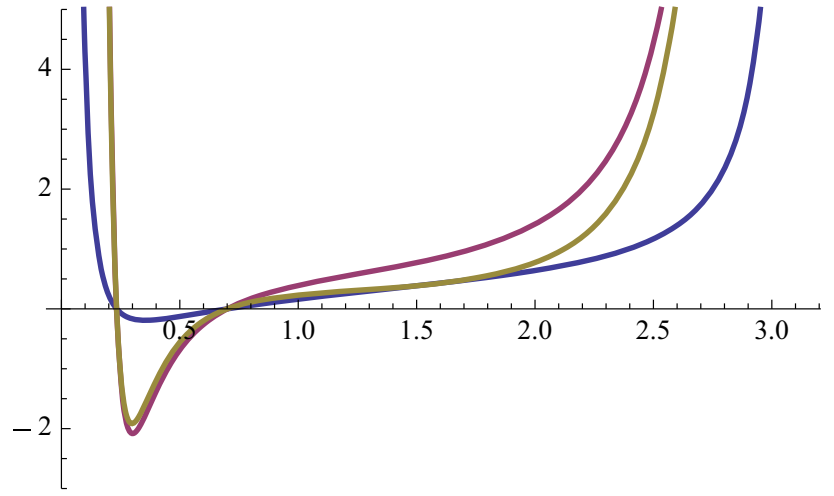

Fig. 4 Plot of effective potential $V(\chi)$ for (i) RN black-hole type solution ( $K=0.9, Q=1, R_{0}=1$ ) for $S_{3}$-I. [ $L=0$ (blue), $L=1$ (red) for timelike and $L=1$ for null.] The shape of the potential shows that the two singularities are not accessible except by the radial $(L=0)$ null geodesic. The geodesic observers can get into the inner region of the black hole. Then they bounce to the outer region in the other copy of the spacetime as usual in the Reisner-Norström geometry in which there exists an infinite tower of spacetime

motions fall into the black hole. However, $V(\chi)$ approaches a constant value as $\chi \rightarrow-\infty$.

For $H_{3}$, the singularity at the center is not accessible except by the radial null geodesic, which is different from the fluidonly case. Similarly to the $S_{3}$-I, it is due the electric charge. When the energy level is low, the geodesic motion is oscillatory as in $S_{3}$-I. When the energy level is high, the geodesic observer can reach the asymptotic infinity. Another interesting solution is dS-type. For this solution, the geodesics escape from the static region crossing the de Sitter-like horizon and reach asymptotic infinity. This is different from the pure de

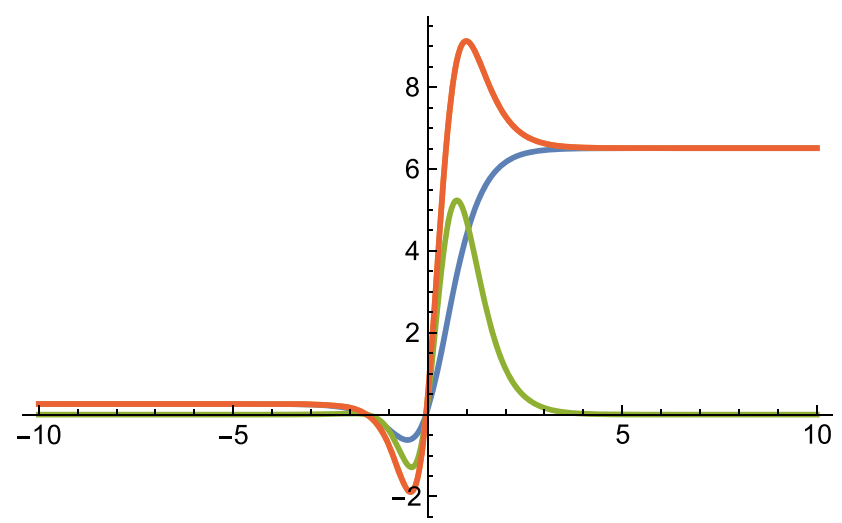

(i)

Fig. 5 Plot of effective potential $V(\chi)$ for $S_{3}$-II. [ $L=0$ (blue), $L=4$ (red) for timelike.] (i) RN black-hole type solution: $K=1, Q=1$, $R_{0}=0.4, L_{\text {null }}=4$. For the low energy level $(\tilde{E})$, the geodesic motion is similar to that of $S_{3}$-I (i), which oscillates in the infinite spacetime tower. For the intermediate energy level, the geodesic motion can reach the inner static region behind the inner horizon. For the high energy
Sitter space in which there can be a stable geodesic motion inside the horizon.

\section{Stability}

In this section, we study the stability of the solutions. We introduce linear spherical scalar perturbations with the metric ansatz,

$d s^{2}=-f(t, \chi) d t^{2}+g(t, \chi) d \chi^{2}+R_{0}^{2} b^{2}(\chi) d \Omega_{2}^{2}$.

The metric perturbations are introduced as

$$
\begin{aligned}
& f(t, \chi)=f_{0}(\chi)+\epsilon f_{1}(t, \chi), \\
& g(t, \chi)=R_{0}^{2}\left[g_{0}(\chi)+\epsilon g_{1}(t, \chi)\right],
\end{aligned}
$$

where $\epsilon$ is a small parameter, and the subscript 0 stands for the background solutions obtained in Sect. 3. Using the function $F(\chi)=8 \pi R_{0}^{2} \rho_{0}(\chi) / 3 s$ defined in Eq. (36), where $\rho_{0}(\chi)$ is the background solution in Table 1, we have

$$
\begin{aligned}
& f_{0}(\chi)=\frac{\rho_{0}(\chi)}{\rho_{c}}=\frac{3 s}{8 \pi R_{0}^{2} \rho_{c}} F(\chi), \\
& g_{0}(\chi)=\frac{1}{F(\chi)} .
\end{aligned}
$$

The contravariant form of the energy-momentum tensor for fluid is written as

$T^{\mu \nu}=(\rho+p) u^{\mu} u^{\nu}+p g^{\mu \nu}$,

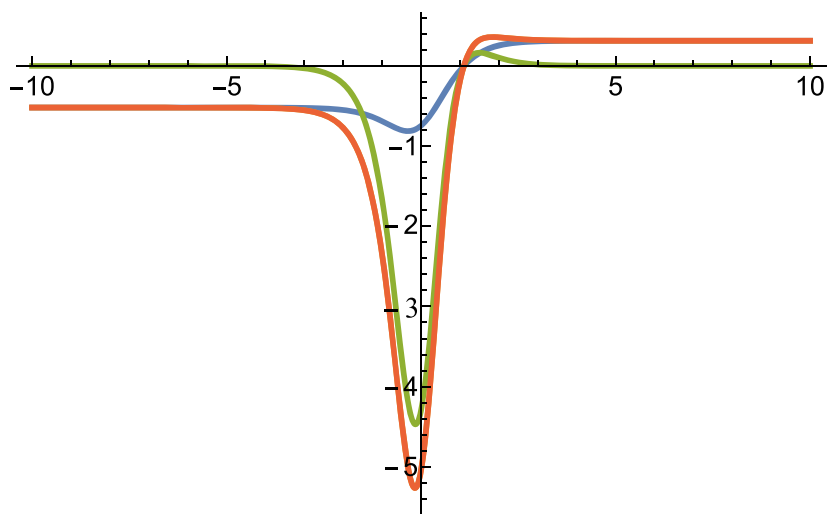

(ii)

level, the geodesic motion can reach the asymptotic infinity at the outer static region. (ii) Schwarzschild black-hole type solution: $K=0.3$, $Q=1, R_{0}=0.6, L_{\text {null }}=4$. The potential is similar to that of the usual Schwarzschild black hole. For the low energy level, the geodesic motion falls into the black hole 


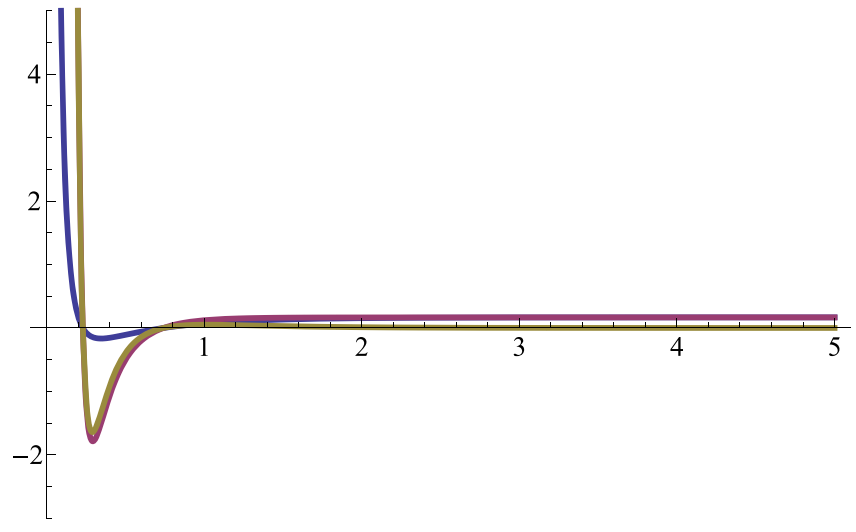

(i)

Fig. 6 Plot of effective potential $V(\chi)$ for $H_{3}$. [ $L=0$ (blue), $L=1$ (red) for timelike.] (i) $\mathrm{RN}$ black-hole type solution: $K=1, Q=1$, $R_{0}=1, L_{\text {null }}=1$. The central singularity is not accessible. For the high energy level, the geodesic motion can reach the asymptotic infin-

with the velocity four-vector

$u^{\mu}=\left[u^{0}(t, \chi), u^{1}(t, \chi), 0,0\right]$.

For the fluid at hand, $p=-\rho / 3$, the perturbations for the energy density and the four-velocity are introduced by

$\rho(t, \chi)=\rho_{0}(\chi)+\epsilon \rho_{1}(t, \chi)$,

$u^{0}(t, \chi)=u_{0}^{0}(\chi)+\epsilon u_{1}^{0}(t, \chi)$,

$u^{1}(t, \chi)=u_{0}^{1}(\chi)+\epsilon u_{1}^{1}(t, \chi)$.

We have $u_{0}^{1}(\chi)=0$ for the comoving background fluid. From the normalization $u^{\mu} u_{\mu}=-1$, we have $u_{0}^{0}(\chi)=1 / \sqrt{f_{0}(\chi)}$ and $u_{1}^{0}(t, \chi)=-f_{1} u_{0}^{0} /\left(2 f_{0}\right)=-f_{1} /\left(2 f_{0}^{3 / 2}\right)$.

For the electric field, we introduce the simplest perturbation along the radial direction only, by which there is no magnetic field induced by the perturbation,

$\mathcal{F}_{t \chi}^{\prime}=-\mathcal{F}_{\chi t}^{\prime}=E(t, \chi)=E_{0}(\chi)+\epsilon E_{1}(t, \chi)$,

where $E_{0}(\chi)$ is given in Eq. (30).

Now we apply the perturbations (44), (45), and (50)-(53), and expand the field equations in the first order of $\epsilon$. From the $(0,1)$ component of the Einstein's equation, we get

$u_{1}^{1}(t, \chi)=-\sqrt{\frac{2 \pi R_{0}^{2} \rho_{c}}{3}} \frac{\dot{g}_{1} b^{\prime} F}{s^{2} b \sqrt{F}}$.

Therefore, the perturbations of the four-vector, $u_{1}^{0}$ and $u_{1}^{1}$ in Eqs. (51) and (52), are expressed by the background functions and the metric perturbations. There are seven equations in total for four perturbations, $f_{1}, g_{1}, \rho_{1}$ and $E_{1}$; three from

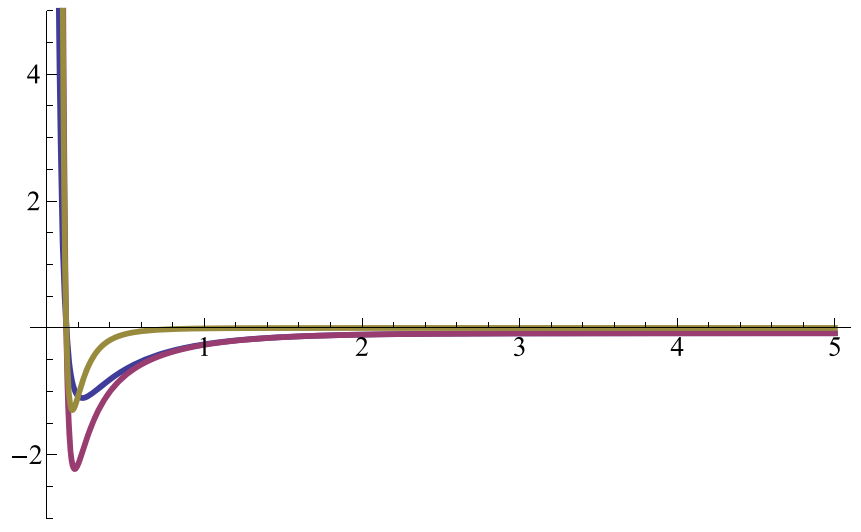

(ii)

ity. (ii) dS-type solution: $K=1.5, Q=1, R_{0}=1, L_{\text {null }}=0.2$. There is no stable geodesic motion inside the de Sitter-like horizon. All the geodesics escape from the static region crossing the horizon

Einstein's equation, two from $\nabla_{\mu} T^{\mu \nu}=0$, and two from $\nabla_{\mu} \mathcal{T}^{\mu \nu}=0$. Four of them are independent equations. After manipulating equations with

$f_{1}(t, \chi)=e^{i \omega t} \psi(\chi)$

$g_{1}(t, \chi)=e^{i \omega t} \varphi(\chi)$,

the equation for $\varphi(\chi)$ is decoupled as

$$
\begin{gathered}
-F^{2} \varphi^{\prime \prime}-\left[3 F F^{\prime}+F^{2}\left(3 \frac{b^{\prime \prime}}{b^{\prime}}+s \frac{b}{b^{\prime}}\right)\right] \varphi^{\prime} \\
+\left[\frac{\omega^{2}}{\sigma}-2 F F^{\prime \prime}-F F^{\prime}\left(4 \frac{b^{\prime \prime}}{b^{\prime}}-\frac{b^{\prime}}{b}-s \frac{b}{b^{\prime}}\right)\right. \\
\left.-2 F^{2}\left(\frac{b^{\prime \prime \prime}}{b^{\prime}}-\frac{b^{\prime 2}}{b^{2}}+s \frac{b b^{\prime \prime}}{b^{\prime 2}}-s\right)\right] \varphi=0,
\end{gathered}
$$

where $\sigma \equiv 1 /\left(8 \pi R_{0}^{4} \rho_{c} s\right)=1 /\left(8 \pi R_{0}^{4}\left|\rho_{c}\right|\right)>0$ for all classes. The coefficients of the above equation depend only on the background functions $F(\chi)$ and $b(\chi)$.

By transforming the radial coordinate and the amplitude function as

$z=\int_{0}^{\chi} \frac{d \chi}{\sqrt{2} F(\chi)}, \quad \Phi(z)=N \frac{F(\chi) b^{\prime}(\chi)}{z} \varphi(\chi)$,

where $N$ is a normalization constant, we get the perturbation equation in the nonrelativistic Schrödinger-type,

$$
\begin{aligned}
& {\left[-\frac{1}{2} \frac{d^{2}}{d z^{2}}-\frac{1}{z} \frac{d}{d z}+U(z)\right] \Phi(z)=-\frac{\omega^{2}}{\sigma} \Phi(z)} \\
& =-8 \pi R_{0}^{4}\left|\rho_{c}\right| \omega^{2} \Phi(z) \equiv \Omega \Phi(z) .
\end{aligned}
$$


The potential is given by

$$
\begin{aligned}
U[z(\chi)]= & F^{2}\left[-\frac{F^{\prime \prime}}{F}+\left(\frac{F^{\prime}}{F}\right)^{2}+\frac{F^{\prime}}{F}\left(\frac{b^{\prime \prime}}{b^{\prime}}+2 \frac{b^{\prime}}{b}+4 s\right)\right. \\
& \left.+2\left(\frac{b^{\prime \prime}}{b^{\prime}}\right)^{2}+s\right],
\end{aligned}
$$

where we used $s b / b^{\prime}=-b^{\prime \prime} / b^{\prime}, b^{\prime \prime \prime} / b^{\prime}=-s$, and $b^{\prime \prime} / b=$ $-s$. Since there always exists a positive eigenvalue $\Omega$ for any type of potential $U$, i.e., $\omega^{2}<0$, this system is unconditionally unstable.

The stability story is very similar to the fluid-only case. When perturbations are introduced to the static fluid, the fluid becomes time dependent, which drives the Universe to undergo the Friedmann expansion. This type of instability does not necessarily mean that the black-hole structure is destroyed. Instead, the instability indicates that the background universe undergoes expansion while the black-hole structure sustains.

When the perturbation of the electric field is considered, the instability can be related with the destruction of the blackhole structure. It is known that the Cauchy (inner) horizon of the charged black hole is unstable to form a singularity [16]. The perturbation introduced in this work may develop such an instability in the RN black-hole type solution.

\section{Conclusions}

We investigated the gravitational field of static fluid plus electric field. Both of the fluid and the electric field are the sources of the gravitational field, but the way to curve the spacetime is a bit different from each other. By adopting the equation of state $p(r)=-\rho(r) / 3$, the fluid is responsible for the topology of the background space. The spatial topology can be either closed $\left(\mathrm{S}_{3}\right)$ or open $\left(\mathrm{H}_{3}\right)$. Such a nature of the spatial topology is not observed everywhere. Instead, the signature of the background spatial topology appears at some place of the spacetime.

Based on the background topology, there exist various types of solutions in three classes which we named as $S_{3}$-I, $\mathrm{S}_{3}$-II, and $H_{3}$. Interesting classes are $\mathrm{S}_{3}$-I and $H_{3}$ although the class $S_{3}$-II has most varieties in solution. The most interesting solutions are the black-hole solutions. Due to the presence of the electric field, the black-hole geometry mimics that of the Reisner-Norström spacetime. This type of black hole exists in both $\mathrm{S}_{3}$ and $\mathrm{H}_{3}$ spaces. (There exists also a Schwarzschildtype black hole in $S_{3}$-II.) The central singularity inside the black hole of this type of solution is due to the electric source as well as the fluid source. There is a naked singularity in $S_{3}$-I at the antipodal point which is not accessible except by the radial null rays. The formation of this singularity is caused by the fluid. The geodesics of the Reisner-Norström black-hole type solution exhibit the oscillatory orbit in the infinite tower of the spacetime encountered in the usual Reisner-Norström geometry.

All the solutions obtained in this paper are unconditionally unstable. This is not surprising because the stability story is similar to the fluid-only case in Ref. [7]. The reason of the instability is that the static fluid becomes unstable (time dependent) with small perturbations and drives the background geometry to the Friedmann expansion. In addition, there is an electric field for which it is well known that the pure charged black-hole solution (Reisner-Norström geometry) is unstable under perturbations.

The solutions investigated in this paper are useful in studying the magnetic monopole in the closed/open space, which is under investigation currently. Usually, the outside geometry of the magnetic monopole is the same with that of the charged black hole (Reisner-Norström geometry) [17-21]. Since we obtained the charged black-hole solution in $\mathrm{S}_{3} / \mathrm{H}_{3}$ with the aid of fluid, it is very interesting to investigate the magnetic monopole in the presence of fluid. It may give rise to insight about the monopole in the closed/open space. The asymptotic geometry of this type of the gauge monopole is worth while to investigate and will be very interesting to compare with the usual monopole geometry. In addition, the removal of the singularity is also a very interesting issue. For the usual case, the monopole field removes the singularity of the charged solution. For this case, however, the formation of the singularity is caused not only by the electric charge, but also by the fluid. It is interesting to see if the monopole field can regularize the singular behavior of the fluid.

Acknowledgements The author is grateful to Hyeong-Chan Kim and Gungwon Kang for useful discussions. This work was supported by the grant from the National Research Foundation funded by the Korean government, no. NRF-2017R1A2B4010738.

Data Availability Statement This manuscript has no associated data or the data will not be deposited. [Authors' comment: No data-set is associated with this study.]

Open Access This article is distributed under the terms of the Creative Commons Attribution 4.0 International License (http://creativecomm ons.org/licenses/by/4.0/), which permits unrestricted use, distribution, and reproduction in any medium, provided you give appropriate credit to the original author(s) and the source, provide a link to the Creative Commons license, and indicate if changes were made. Funded by SCOAP ${ }^{3}$.

\section{References}

1. P.A.R. Ade et al. [Planck Collaboration], Astron. Astrophys. 594, A13 (2016). https://doi.org/10.1051/0004-6361/201525830. arXiv:1502.01589 [astro-ph.CO] 
2. G.F.R. Ellis, R. Maartens, Class. Quantum Gravity 21, 223 (2004). https://doi.org/10.1088/0264-9381/21/1/015. arXiv:gr-qc/0211082

3. G.F.R. Ellis, J. Murugan, C.G. Tsagas, Class. Quantum Gravity 21(1), 233 (2004). https://doi.org/10.1088/0264-9381/21/1/016. arXiv:gr-qc/0307112

4. P. Labrana, Phys. Rev. D 91(8), 083534 (2015). https://doi.org/10. 1103/PhysRevD.91.083534. arXiv:1312.6877 [astro-ph.CO]

5. M. Bucher, A.S. Goldhaber, N. Turok, Phys. Rev. D 52, 3314 (1995). https://doi.org/10.1103/PhysRevD.52.3314. arXiv:hep-ph/9411206

6. J. White, Y 1 Zhang, M. Sasaki, Phys. Rev. D 90(8), 083517 (2014). https://doi.org/10.1103/PhysRevD.90.083517. arXiv:1407.5816 [astro-ph.CO]

7. I. Cho, H.C. Kim, Phys. Rev. D 95(8), 084052 (2017). https://doi. org/10.1103/PhysRevD.95.084052. arXiv:1610.04087 [gr-qc]

8. J.D. Bekenstein, Phys. Rev. D 4, 2185 (1971). https://doi.org/10. 1103/PhysRevD.4.2185

9. R.D. Sorkin, R.M. Wald, Z.J. Zhang, Gen. Relativ. Gravit. 13, 1127 (1981). https://doi.org/10.1007/BF00759862

10. A. Pesci, Class. Quantum Gravity 24, 2283 (2007). https://doi.org/ 10.1088/0264-9381/24/9/009. arXiv:gr-qc/0611103

11. I. Semiz, Rev. Math. Phys. 23, 865 (2011). https://doi.org/10.1142/ S0129055X1100445X. arXiv:0810.0634 [gr-qc]
12. K. Lake, Phys. Rev. D 67, 104015 (2003). https://doi.org/10.1103/ PhysRevD.67.104015. arXiv:gr-qc/0209104

13. K.A. Bronnikov, O.B. Zaslavskii, Phys. Rev. D 78, 021501 (2008). https://doi.org/10.1103/PhysRevD.78.021501。 arXiv:0801.0889 [gr-qc]

14. I. Cho, H.C. Kim, arXiv:1703.01103 [gr-qc]

15. C.W. Misner, D.H. Sharp, Phys. Rev. 136, B571 (1964). https:// doi.org/10.1103/PhysRev.136.B571

16. Y. Gursel, V.D. Sandberg, I.D. Novikov, A.A. Starobinsky, Phys. Rev. D 19, 413 (1979). https://doi.org/10.1103/PhysRevD.19.413

17. G.W. Gibbons, Lect. Notes Phys. 383, 110 (1991). https://doi.org/ 10.1007/3-540-54293-0_24. arXiv:1109.3538 [gr-qc]

18. Y.M. Cho, P.G.O. Freund, Phys. Rev. D 12, 1588 (1975). Erratum: [Phys. Rev. D 13, 531 (1976)]. https://doi.org/10.1103/PhysRevD. 13.531.2. https://doi.org/10.1103/PhysRevD.12.1588

19. F.A. Bais, R.J. Russell, Phys. Rev. D 11, 2692 (1975). Erratum: [Phys. Rev. D 12, 3368 (1975)]. https://doi.org/10.1103/ PhysRevD.12.3368.2. https://doi.org/10.1103/PhysRevD.11.2692

20. P.B. Yasskin, Phys. Rev. D 12, 2212 (1975). https://doi.org/10. 1103/PhysRevD.12.2212

21. P. Cordero, C. Teitelboim, Ann. Phys. 100, 607 (1976). https://doi org/10.1016/0003-4916(76)90074-9 